\title{
AVANT-PROPOS
}

Ce livre de biochimie appliquée est une réédition revue et augmentée du précédent ouvrage paru en 2009, mais cette fois il est associé à un site WEB dédié. Comme le précédent, il doit :

$\triangleright$ permettre au lecteur d'acquérir les connaissances les plus récentes sur les substances et les molécules naturelles exploitées par l'homme ;

$\triangleright$ faire le lien entre la biochimie des substances naturelles et leurs applications dans des domaines variés comme l'agriculture, l'environnement, la production d'énergie, l'alimentation animale et humaine, la santé et de nombreux autres domaines plus proches du monde industriel (papeterie, cosmétique, agro-alimentaire, pharmacie, chimie...).

L'ouvrage est maintenant divisé en 6 parties et en 19 chapitres. Trois nouveaux chapitres ont été ajoutés: le premier, portant sur la gélatine (chap. 12), complète la partie III sur les substances d'origine animale, le deuxième, sur les polymères microbiens (chap. 15), abonde la partie IV sur la biochimie des substances d'origine microbienne et le troisième, sur les extrêmozymes (chap. 18), enrichit la partie $V$ sur les applications de l'enzymologie. Le chapitre 8 , sur les métabolites des microalgues et des cyanobactéries, a été complètement revu et mis à jour. D'autres données récentes ont été ajoutées ou actualisées, comme la glycomique dans le chapitre 1, la séparation et l'identification des lipides dans le chapitre 3, I'hémoglobine en biochimie médicale dans le chapitre 9 et les cultures de cellules animales dans le chapitre 19.

- Plus globalement, la première partie est consacrée aux substances issues du règne végétal (plantes terrestres). En effet, les plantes fournissent un nombre considérable de molécules comme les glucides, les protides, les corps gras, les pigments, les arômes et une grande partie des substances actives des médicaments. Celles-ci sont de nature à intéresser toutes sortes d'activités industrielles, pharmaceutique, agro-alimentaire, cosmétique... La pharmacopée traditionnelle a toujours exploité les «vertus» des plantes médicinales, sans toujours en connaître les principes actifs. La plupart de ces biomolécules sont maintenant bien identifiées et, par synthèse chimique, on est arrivé à en faire des "copies», souvent améliorées. Aujourd'hui, les substances naturelles sont privilégiées dans l'optique d'une utilisation raisonnée et durable des produits de la biosphère. Si les trois premiers chapitres traitent classiquement des glucides, des protides et des lipides végétaux, dans les trois chapitres suivants une attention particulière est portée aux huiles essentielles, à la lignine et aux lectines dont les applications sont de plus en plus nombreuses.

- La deuxième partie, divisée en deux chapitres traite, d'une part, des polysaccharides des parois des algues et de leurs applications, dominées par le secteur de l'alimentation 
et de la santé, et d'autre part, des métabolites issus des microalgues et des cyanobactéries. Les potentialités d'utilisation des végétaux ou des biomolécules qui en sont extraites, purifiées et/ou modifiées par voie enzymatique et chimique, les font «sortir » du domaine agro-alimentaire vers d'autres nouveaux secteurs industriels comme la production de biocarburants. Les méthodes d'étude (procédés d'extraction, techniques de purification, de séparation et de dosage) sont également présentées.

- La valorisation des substances d'origine animale est passée en revue dans la troisième partie. Il s'agit notamment des dérivés sanguins, des sous-produits de l'industrie du lait et du fromage (lactosérum), des ovoproduits.

- Les produits issus des micro-organismes, représentés principalement par les protéines, les antibiotiques sont abordés dans la quatrième partie.

- L'utilisation des enzymes, secteur en plein essor, fait l'objet de la partie suivante. En raison de leurs rôles et de leurs applications technologiques bien caractérisées, les enzymes font l'objet d'études intensives et leurs modalités d'action sont de mieux en mieux connues. Par ailleurs, l'obtention de produits de qualité optimale suppose une bonne maîtrise de l'action de ces enzymes par la connaissance, non seulement de leur fonctionnement, mais aussi de l'influence de certains effecteurs physico-chimiques comme les conditions d'utilisation (température, $\mathrm{pH}$, présence d'interférants...). Des procédés industriels basés totalement ou partiellement sur les enzymes ont été mis au point. Les enzymes sont devenues, de ce fait, des auxiliaires technologiques incontournables. Les répercussions sur les plans économique et environnemental ne sont plus à démontrer. Le génie génétique, le génie enzymatique, le génie métabolique aidant, il est actuellement possible de produire des souches de micro-organismes fabriquant des enzymes capables d'opérer dans les conditions très spécifiques de l'environnement industriel et/ou de synthétiser des métabolites originaux. Dans cette cinquième partie les enzymes immobilisées, leurs différentes applications dans la transformation des matériaux biologiques et leur utilisation de plus en plus fréquente en bio-analyse sont présentées. Les récents progrès dans le domaine des capteurs enzymatiques sont aussi passés en revue de même que leurs utilisations actuelles.

- Enfin, en raison de leur exploitation récente dans l'industrie et des perspectives économiques importantes, actuelles et à venir, les cultures de cellules végétales et animales, leurs contraintes et leurs applications sont présentées dans la dernière partie.

- Etant donné les développements très rapides et les innovations techniques continues dans le domaine de la biochimie appliquée, les références bibliographiques deviennent rapidement obsolètes. Nous avons d'abord essayé, dans la mesure du possible, d'intégrer les références les plus actuelles et les plus accessibles pour les étudiants, les lecteurs souhaitant trouver plus d'informations pourront consulter une webographie sur le site dédié.

- Le glossaire reprenant les définitions des principaux termes et concepts faisant l'objet du livre, largement abondé, reste placé à la fin de l'ouvrage papier pour en faciliter la consultation; comme précédemment les termes en gras présent dans l'ouvrage sont définis dans ce glossaire.

- Un index des termes utilisés et des espèces citées dans le texte complète le livre. 
Cet ouvrage, caractérisé par un traitement plus conséquent des métabolites du règne végétal, se démarque de la majorité des ouvrages de biochimie disponibles actuellement où très souvent le végétal est un peu le laissé pour compte, avec parfois un petit chapitre sur la photosynthèse et la biochimie spécifique du chloroplaste en négligeant le métabolisme secondaire (très important dans le règne végétal). Pour beaucoup, la biochimie est essentiellement animale, voire humaine, et cet ouvrage devrait leur ouvrir de nouveaux horizons. Ainsi, c'est volontairement que nous mettons en avant la biochimie du végétal et les nombreuses substances qui en sont extraites, pour certaines produites et, dans tous les cas, abondamment et depuis longtemps utilisées par l'homme.

Ce travail vient en complément de la biochimie fondamentale traitée dans d'autres ouvrages de la même collection et s'adresse principalement aux étudiants des cursus scientifiques (sciences de la nature et du vivant, écoles d'ingénieurs...), aux étudiants des licences et masters de biologie, de biochimie, de biotechnologie, aux étudiants des IUT et des BTS à orientation biologique et biochimique, aux étudiants des nombreuses et nouvelles licences professionnelles dont une partie de la formation porte sur les applications de la biologie et de la biochimie, enfin aux cadres et aux chercheurs des industries agro-alimentaires et des sciences pharmaceutiques.

La formation de ces lecteurs potentiels étant très disparate, nous avons volontairement placé au début de chaque chapitre quelques rappels des données générales de biochimie structurale et/ou métabolique indispensables pour comprendre la suite du document (évitant ainsi au lecteur le recours à un autre ouvrage plus général de biochimie). Toutefois quelques notions de base en biochimie structurale et métabolique sont malgré tout nécessaires, voire indispensables, pour aborder, sans peine, certaines parties de cet ouvrage. Par contre, certains sujets traités sont souvent difficiles d'accès, particulièrement en langue française. De ce fait, nous espérons que cet ouvrage comblera le manque dans ce domaine.

\section{MODE D'EMPLOI}

Le présent livre est un abrégé. Il est conçu pour diverses utilisations par le lecteur.

C'est un ouvrage qu'il faut cette fois consulter en ayant à sa disposition, soit un ordinateur, soit une tablette, voire même un smartphone, connecté à Internet.

En effet, les encarts sont maintenant sur le site WEB dédié, mais des renvois sont placés dans le texte pour signaler l'existence de ces compléments d'information. Plusieurs nouveaux encarts correspondant aux différents chapitres ont été placés sur le site dédié : les tanins, I'huile d'olive, une huile aux multiples vertus, l'huile d'argan, les oméga 3, la biotechnologie des microalgues et son développement, les principales caractéristiques des grands groupes taxonomiques de microalgues et de Cyanobactéries, une hémoglobine extraite de vers marins, le sang artificiel, les anticoagulants du futur, le lait maternel, les exopolysaccharides des bactéries lactiques, I'histoire de la gélatine, l'histoire des enzymes, les parfums, les compléments alimentaires. Les encarts précédemment présents dans l'ouvrage ont été transférés sur le site après mise à jour lorsque cela a été nécessaire. 
Il s'agit de : le pouvoir sucrant, les interdépendances métaboliques dans le diabète, les fibres alimentaires, la maladie cœliaque, la réaction de Maillard, Agrobacterium, la stratégie de I'ARN antisens, le marquage radioactif en biochimie, la discrimination isotopique, I'intolérance au lactose, la résistance aux antibiotiques, les puces à ADN.

Le lexique français-anglais est lui aussi en ligne ainsi que les annexes portant sur les codes EC des enzymes et les additifs alimentaires cités dans le texte ainsi que sur les principaux groupes caractéristiques rencontrés dans les molécules organiques.

Enfin, la section d'autoévaluation pour chacun des chapitres est proposée de façon plus ludique au niveau du site WEB. Un accès aux réponses des questions à réponses ouvertes courtes (QROC) est proposé sous forme de mots ou de phrase clefs et les réponses au QCM sont elles aussi fournies. Elle est destinée à aider les étudiants à contrôler l'acquisition de leurs connaissances afin de mieux préparer leurs évaluations.

Comme dans la précédente édition, une lecture au fil du texte permettra d'acquérir une vision générale de la biochimie appliquée. Des rappels de biochimie structurale ou métabolique au début de chaque chapitre définissent les prérequis.

Un certain nombre d'éléments sont disponibles en appui pour le lecteur : liste d'abréviations, de symboles et acronymes; glossaire de définition des principaux termes et concepts ; index de mots et d'espèces ; compléments d'information et annexes supplémentaires sur le site web.

Les outils précédents sont proposés pour une consultation ultérieure de l'ouvrage, ponctuelle ou plus structurée. Par exemple, un lecteur concerné par une technique et/ou une application trouvera dans l'Abrégé une première série d'informations qu'il pourra compléter lors de recherches plus poussées dans les ouvrages cités dans la bibliographie ou dans d'autres ouvrages. Si son étude ultérieure utilise le web, la webographie fournie et le lexique anglais-français lui feront gagner un temps précieux.

De nombreux appareils ou instruments couramment utilisés dans les laboratoires sont cités dans cet ouvrage; il est possible à chaque fois de trouver un complément d'information dans le précédent ouvrage des mêmes auteurs, dans la même collection, Mémento technique à l'usage des biologistes et des biochimistes.

\section{REMERCIEMENTS}

Les auteurs remercient vivement les membres du comité d'édition qui, par leurs remarques et leur conseils, ont permis d'améliorer cette réédition et tout particulièrement $M$. Guy Hervé qui a revu l'ensemble avec beaucoup d'attention. C'est ensuite avec plaisir qu'ils remercient ici Monsieur Jean Bornarel, qui leur a proposé de rééditer cet ouvrage, et ses proches collaboratrices avec lesquelles ils ont toujours eu d'excellents contacts par mail ou par téléphone et dont l'efficacité et les compétences ne sont plus à prouver : Madame Laura Capolo et Mesdames Julie Ridard et Valérie Szczupal (pour la première édition) et Sylvie Bordage (pour cette nouvelle édition) ainsi que Anne-Laure Passavant pour l'élaboration du site web dédié. 
Aderrazak Marouf remercie particulièrement Boucherit Kebir, professeur de biochimie au Centre Universitaire d'Ain Temouchent et Abdelkrim Cheriti, professeur de chimie à I'Université de Béchar, pour la relecture de certaines parties de l'ouvrage, ainsi que tous ses collègues biologistes et chimistes de l'Université d'Oran qui ont apporté leur grain de sel à ce travail par leurs critiques ou leurs suggestions. Il tient, enfin, à remercier son épouse dont la patience a été déjà mise à dure épreuve durant l'élaboration de la première édition et qui, loin de fléchir, a su aussi l'encourager et l'aider, par ses sacrifices incessants, tout au long des péripéties de cette deuxième édition.

Gérard Tremblin tient à remercier l'ensemble de ses collègues de l'équipe MMS (Mer, Molécules, Santé) qui, en continuant à l'accueillir en tant que professeur émérite dans les locaux de l'Université du Maine, lui permettent de poursuivre, entre autres, son activité de rédacteur d'ouvrages scientifiques. Un remerciement tout particulier au professeur Annick Morant-Manceau qui l'accueille dans son bureau et dans lequel il essaie de se faire tout petit et discret (ce qui n'est pas toujours facile). Il tient aussi à remercier son collègue, le professeur et coauteur Abderrazak Marouf qui lui a proposé, il y a une dizaine d'année, de collaborer à cet ouvrage, lui ouvrant ainsi des perspectives d'activités de fin de carrière qu'il n'avait pas envisagées. Cette collaboration a été fructueuse car, en dehors de cette réédition, un ouvrage commun est paru en 2013, Mémento technique à l'usage des biologistes et des biochimistes, et un autre est en préparation. Il tient enfin à remercier son épouse et ses cinq garçons à qui il dédie ce travail bien qu'aucun ne se soit intéressé à la Biologie et à la Biochimie et qui, de ce fait, ne liront pas cet ouvrage. 
7 KVSDJHIQMQMRQDCIOHEDQN 